\title{
Submitted: Diagnostic value of shear wave velocity \\ Accepted: in polycystic ovarian syndrome
} 20.10.2021

Published: 29.11 .2021

Keywords

acoustic radiation

force impulse imaging; elastography; polycystic ovarian syndrome; shear wave velocity; ultrasonography

\author{
Turkan Gursu'1, Halime Cevik², Güldeniz Aksan Desteli ${ }^{3}$, \\ Birnur Yilmaz², Tevfik Berk Bildaci³ ${ }^{3}$ Alper Eraslan ${ }^{4}$
}

${ }^{1}$ Department of Obstetrics and Gynecology, University of Health Sciences Turkey Zeynep Kamil Maternity and Children's Training And Research Hospital, Istanbul, Turkey, Turkey

${ }^{2}$ Department of Radiology, Okan University Hospital, Istanbul, Turkey, Turkey

${ }^{3}$ Department of Obstetrics and Gynecology, Baskent University Istanbul Hospital, Istanbul, Turkey, Turkey

${ }^{4}$ Department of Obstetrics and Gynecology, Reproductive Endocrinology Division, Dünya IVF Center, Kyrenia, Cyprus, Turkey

Correspondence: Turkan Gursu, Department of Obstetrics and Gynecology, University of Health Sciences Turkey Zeynep Kamil Maternity and Children's Training And Research Hospital, Istanbul, Turkey, Acıbadem Istanbul, 34660, Istanbul, Turkey;

e-mail:turkangursu@hotmail.com

DOI: 10.15557/JoU.2021.0047

\begin{abstract}
Aim: In polycystic ovarian syndrome, the ovaries become stiffer due to chronic anovulation. We aimed to compare tissue elasticity in terms of shear wave velocities measured using acoustic radiation force impulse imaging technique between the ovaries of polycystic ovarian syndrome women and non-polycystic ovarian syndrome women. Material and methods: The study was designed as a retrospective data analysis of women who underwent transvaginal ultrasound and acoustic radiation force impulse imaging in a university hospital between July 2014 and March 2015, for various reasons. There were 32 polycystic ovarian syndrome patients and 32 patients without a diagnosis of polycystic ovarian syndrome. Age, body mass index, fasting glucose levels, cycle day 3 follicle stimulating hormone, luteinizing hormone, thyroid stimulating hormone, prolactin, antimullerian hormone levels, and menstrual patterns with clinical hyperandrogenism were evaluated. On the menstrual cycle days $2-4$, by performing a transvaginal ultrasound scan, the ovarian volumes and antral follicle counts in both ovaries were recorded for each woman. The ultrasound system was converted into the elastography mode, and acoustic radiation force impulse imaging was performed. Shear wave velocity $(\mathrm{m} / \mathrm{sec})$ was measured at least 5 times for each ovary, and the mean value was calculated for each polycystic ovarian syndrome and non-polycystic ovarian syndrome woman. Results: Age, body mass index, fasting glucose levels, cycle day 3 follicle stimulating hormone, luteinizing hormone, thyroid stimulating hormone, and prolactin levels were similar between the groups $(p>0,05)$. Antimullerian hormone levels, antral follicle counts, and mean ovarian volumes were statistically different between the groups $(p<0,05)$. Mean shear wave velocity values for both ovaries were $2.12 \pm 0.82(0.78-4.9) \mathrm{m} / \mathrm{sec}$ in the polycystic ovarian syndrome group, and $1.18 \pm 0.41(0.77-2.0) \mathrm{m} / \mathrm{sec}$ in the non-polycystic ovarian syndrome group, which was statistically significantly different $(p=0.016)$. Conclusion: In our study, we found significantly higher shear wave velocity levels in polycystic ovarian syndrome women than non-polycystic ovarian syndrome women, which indicates an impact of the condition on shear wave velocity. The increased acoustic frequencies cause a decreased response in time to transition, and motion becomes out of phase; in other words, scattered waves are faster in stiffer ovaries. Our results are thus compatible with the pathophysiology of the disease. Shear wave velocity is a beneficial tool for evaluating ovarian elasticity in polycystic ovarian syndrome patients in whom the levels are found to be significantly higher than non-polycystic ovarian syndrome women. In light of these findings, shear wave velocity is expected to be slower than polycystic ovarian syndrome levels in ovulatory women.
\end{abstract}




\section{Introduction}

Elastography is a useful tool providing information on tissues by converting stiffness values into an anatomically significant map. Sonoelastography is an ultrasound (US) imaging technique. When the tissue is harder, the sound waves move faster, and their speed provides information about tissue hardness ${ }^{(1)}$. Both qualitative and quantitative types of elastography are available. Acoustic radiation force impulse imaging (ARFI) is a recently developed noninvasive dynamic tissue imaging technique of quantitative elastography. ARFI correlates tissue flexibility with conventional gray-scale images obtained by US. Shear wave velocity (SWV) is measured by ARFI to establish the stiffness of tissues in meters per seconds $(\mathrm{m} / \mathrm{sec})$. With gradually increasing acoustic frequencies, tissues fail to respond in time to the transitions between positive and negative pressures, and the motion becomes out of phase with the acoustic wave frequency. As a result, energy is deposited in the tissues, which causes momentum transfer and an increase in tissue temperature. The resulting displacement of tissue is detected and used for obtaining additional information beyond B-mode imaging ${ }^{(2)}$.

Changes in tissue elasticity are a useful tool used for identifying fibrosis and differentiating between malignant and benign lesions in the tissues of the liver, thyroid, and breast $\mathrm{t}^{(3)}$. Tissue elasticity measured by ARFI and SWV is used in gynecologic and obstetric practice, e.g. in placental tissues, endometrium, myometrium, and cervix ${ }^{(4-6)}$.

Polycystic ovaries are seen in polycystic ovarian syndrome (PCOS) and sometimes also in normally ovulating women (polycystic ovarian morphology). PCOS is diagnosed using the Rotterdam Criteria, based on the presence of at least two criteria of the total three, provided that other etiologies are ruled out $^{(7)}$. One of the criteria is having $\geq 12$ antral follicles (follicles of $2-9 \mathrm{~mm}$ ) or $\geq 10 \mathrm{ml}$ ovarian volume in one or both ovaries.

Stein and Leventhal were the first to report this syndrome, describing the ovaries as rubbery and naming the condition "hyperthecosis" to reflect chronic anovulation ${ }^{(8)}$.

Chronic anovulation is a vicious cycle with stromal tissue producing more androgens, leading to follicles to rest in the antral stage, not progressing to the preantral stage, or not being able to gain dominance due to hypoestrogenic inner environment.

We conducted this study to gain insights into the elasticity pattern and SWV in PCOS ovaries. The primary outcome measure was to compare SWV values between PCOS and non-PCOS women. The secondary outcome measure was to compare the menstruation patterns, clinical properties, and laboratory and other ultrasonographic parameters between PCOS and non-PCOS women.

\section{Material and methods}

The study was designed as a retrospective data analysis of women who underwent vaginal US and ARFI in a university hospital between July 2014 and March 2015, for various reasons. There were 32 patients diagnosed with PCOS based on the Rotterdam Criteria. Also, there were 32 age-matched normally ovulating women having less than 12 antral follicle count per ovaries, selected as the non-PCOS group. For the diagnosis of PCOS, the Rotterdam Criteria were used, comprising: i) menstrual abnormalities such as oligomenorrhea or amenorrhea, ii) clinical or biochemical hyperandrogenism, and iii) ovaries having $\geq 12$ antral follicles (counted with transvaginal US probe resolution of $<8 \mathrm{MHz}$ ) or $\geq 10 \mathrm{ml}$ ovarian volume. Having at least 2 of these 3 criteria is considered to be indicative of $\operatorname{PCOS}^{(7)}$.

Menstruation patterns were recorded as normal menstruation (menstruation/21-35 days), polymenorrhea (menstruation more frequent than 21 days), oligomenorrhea (less frequent than 45 days, or less than 8 cycles per year) or amenorrhea either primary (no menstruation until 17 years of age) or secondary (no menstruation for more than 3 months in a woman who previously had menstruation) $)^{(9)}$.

Clinical hyperandrogenism was defined as having acne or hirsutism. Hirsutism is evaluated with the modified Ferriman-Gallwey (mF-G) scores; $\geq 8$ is considered as clinical hirsutism ${ }^{(10)}$.

Age, body mass index, fasting blood glucose, follicle stimulating hormone (FSH), luteinizing hormone ( $\mathrm{LH})$, thyroid stimulating hormone (TSH), prolactin (PRL), free testosterone (fTest), antimullerian hormone (AMH), dehydroepiandrostenedione-sulfate (DHEA-S), androstenedione, and free androgen index levels were measured at the beginning of the menstrual cycle; and days 2-4 in women having menses. In women with secondary amenorrhea, menstruation was maintained with 5 days of oral medroxyprogesterone acetate (10 mg daily).

We excluded women with abnormal thyroid function tests, elevated prolactin levels, very high DHEA-S levels (>700 pgr/dl), and high 17-hydroxyprogesterone levels.

\section{Ultrasound and elastography technique}

On days 2-4 of the menstruation cycle, all women were evaluated using the conventional gray-scale US, and ovarian volumes were calculated for each patient by measuring the largest dimensions in the antero-posterior, transverse and longitudinal planes of both ovaries $(0.5 \times$ length $\times$ width $\times$ thickness), and the total number of antral follicles (2 to $9 \mathrm{~mm}$ in diameter) was calculated. Peak systolic velocity (PSV) and resistance index (RI) of both ovaries were measured by spectral Doppler imaging. The system was converted into the elastography mode, and ARFI was performed. Each patient was asked to lie supine and hold her breath. The US probe was placed transvaginally for both ovaries, and the examination was performed in the vein-free area with a region of interest of $1 \times 0.5 \mathrm{~cm}$, with depth set at $6 \mathrm{~cm}$. $\mathrm{SWV}(\mathrm{m} / \mathrm{sec})$ was measured at least 5 times for each ovary, and the mean value was calculated in all women. For each woman, the mean SWV of the left ovary was calculated, and the mean SWV of the right ovary was calculated; in the 
Tab. 1. General parameters of patients in the PCOS and non-PCOS groups

\begin{tabular}{|c|c|c|c|}
\hline Age & $\begin{array}{c}\text { PCOS } \\
(\boldsymbol{n}=\mathbf{3 2})\end{array}$ & $\begin{array}{c}\text { non-PCOS } \\
(\boldsymbol{n}=\mathbf{3 2})\end{array}$ & $\boldsymbol{p}$ \\
\hline BMI (kg/m²) & $\begin{array}{c}20.55 \pm 4.86 \\
(18-35)\end{array}$ & $\begin{array}{c}24.7 \pm 3.67 \\
(18-31)\end{array}$ & 0.21 \\
\hline $\begin{array}{c}\text { Fasting glucose } \\
(\mathrm{mg} / \mathrm{dl})\end{array}$ & $\begin{array}{c}96 \pm 11 \\
(79-101)\end{array}$ & $\begin{array}{c}21.8 \pm 1.54 \\
(18-26)\end{array}$ & 0.45 \\
\hline $\begin{array}{c}\mathrm{TSH} \\
(\mu \mathrm{IU} / \mathrm{mL})\end{array}$ & $\begin{array}{c}3.11 \pm 1.98 \\
(1.7-4.06)\end{array}$ & $\begin{array}{c}2.26 \pm 1.03 \\
(1.43-4.12)\end{array}$ & 0.21 \\
\hline $\begin{array}{c}\mathrm{PRL} \\
(\mu \mathrm{g} / \mathrm{l})\end{array}$ & $\begin{array}{c}28 \pm 5.32 \\
(22-38)\end{array}$ & $\begin{array}{c}25 \pm 4.78 \\
(17-34)\end{array}$ & 0.11 \\
\hline $\begin{array}{c}\mathrm{FSH} \\
(\mathrm{mIU} / \mathrm{mL})\end{array}$ & $6.70 \pm 1.23(4-9)$ & $6.2 \pm 1.21(5-10)$ & 0.23 \\
\hline $\begin{array}{c}\mathrm{LH} \\
(\mathrm{IU} / \mathrm{L})\end{array}$ & $\begin{array}{c}8.67 \pm 2.05 \\
(6-13)\end{array}$ & $\begin{array}{c}6.7 \pm 1.76 \\
(4.5-12)\end{array}$ & 0.16 \\
\hline $\mathrm{BM}$ &
\end{tabular}

$\mathrm{BMI}$ - body mass index; TSH - thyroid stimulating hormone; PRL - prolactin; FSH - follicle stimulating hormone; LH - luteinizing hormone

next step, the mean ovarian SWV was calculated by adding the mean left and the mean right SWV values and dividing the result by two (SWV = left ovarian SWV + right ovarian SWV/2). ARFI (Acuson S3000TM Ultrasound System, Siemens Health Care, Mountain View, Ca, USA) imaging was routinely performed along with conventional US of the ovaries in all cases. The gray-scale US and ARFI studies were performed using a transvaginal $8 \mathrm{MHz}$ probe.

We excluded women with any cystic or solid mass in the ovaries on days 2-4 of the menstrual cycle detected via US. The data obtained from PCOS women and non-PCOS women were evaluated and compared.

\section{Statistical analysis}

Statistical analysis was performed using the SPSS version 22 (Statistical Program for Social Sciences, IBM, Chicago, IL). Demographic continuous data were characterized by means and standard deviations (SD), and nominal variables were expressed as percentages. The Shapiro-Wilk test was used for assessing normality. All continuous variables were found to be normally distributed. Parametric tests were used for variables with normal distribution. Differences in mean values were analyzed with Student's t-test. The characteristics between groups were compared with chisquare test. A $p$ value $\leq 0.05$ was considered as statistically significant.

\section{Results}

The study comprised 32 patients in the PCOS group, and 32 women in the non-PCOS group. Age, body mass index, fasting glucose levels, and hormone levels on days 2-4 of the menstrual cycle (FSH, LH, and TSH and PRL) are shown in Tab. 1. There were no statistically significant differences among these parameters in both groups ( $p=$ nonsignificant (NS)) (Tab. 1).

The menstruation parameters were significantly different between the PCOS and non-PCOS women. There were 8 $(25 \%)$ normally menstruating women in the PCOS group, but there were 29 (90.6\%) normally menstruating women in the non-PCOS group $(p=$ significant $(\mathrm{S}))$. Oligomenorrhea was seen in $23(71.87 \%)$ patients in the PCOS group and in $2(6.25 \%)$ women in the non-PCOS group $(p=\mathrm{S})$. All PCOS patients had clinical hyperandrogenism (100\%); in $12(18.75 \%)$ women, both acne and hirsutism were seen, in $2(6.25 \%)$ patients only hirsutism was present, and in 18 $(56.25 \%)$ only acne was observed. In the non-PCOS group, there were $6(18.75 \%)$ patients with clinical hyperandrogenism. The difference was statistically significant $(p=\mathbf{S})$. Biochemical hyperandrogenism was seen in 28 patients $(87.5 \%)$ in the PCOS group, and in 2 patients in the nonPCOS group (6.25\%). AMH levels were statistically higher among the PCOS women than in the non-PCOS group $(p=\mathrm{S})$ (Tab. 2). AFC levels were statistically higher in the PCOS than in the non-PCOS group $(p=\mathrm{S})$. Mean ovarian volumes were statistically different between the two groups $(p=\mathrm{S})$. Peak systolic velocities and resistance indices were statistically different between the PCOS and control groups $(p=$

Tab. 2. Features of menstruation and hyperandrogenism in PCOS and non-PCOS women

\begin{tabular}{|c|c|c|c|c|}
\hline & & $\begin{array}{l}\text { PCOS } \\
n=32\end{array}$ & $\begin{array}{c}\text { Non-PCOS } \\
n=32\end{array}$ & $p$ \\
\hline \multicolumn{2}{|c|}{$\begin{array}{l}\text { Normal menstruation } \\
\text { (1 in } 21-35 \text { days) }\end{array}$} & 8 & 29 & 0.000 \\
\hline \multicolumn{2}{|c|}{ Polymenorrhea more frequent than 21 days } & 0 & 1 & 0.634 \\
\hline \multicolumn{2}{|c|}{$\begin{array}{c}\text { Oligomenorrhea } \\
\text { (less frequent than } 45 \text { days or Less than } 8 \text { menses per one year) }\end{array}$} & 23 & 2 & 0.000 \\
\hline \multicolumn{2}{|c|}{ Secondary amenorrhea } & 1 & 0 & 0.634 \\
\hline \multirow{2}{*}{ Clinical hyperandrogenism } & $\begin{array}{l}\text { Hirsutism } \\
\text { (Modified Ferriman Gallwey (mF-G) score } \geq 8 \text { ) }\end{array}$ & 17 & 2 & 0.010 \\
\hline & Acne vulgaris & 20 & 4 & 0.000 \\
\hline \multirow{4}{*}{ Biochemical hyperandrogenism } & DHEA-S (ng/dl) & $329.97 \pm 149.93(98-560)$ & $249.71 \pm 82.29(86-320)$ & 0.010 \\
\hline & Free testosterone $(\mathrm{ng} / \mathrm{dl})$ & $812 \pm 235(400-1400)$ & $420 \pm 147(300-1200)$ & 0.000 \\
\hline & Androstenedione $(\mathrm{ng} / \mathrm{ml})$ & $5.12 \pm 1.04(0.4-5.12)$ & $3,12 \pm 0,98(0,2-3,98)$ & 0.06 \\
\hline & Free Androgen Index & $4.81 \pm 2.03(3.15-7.01)$ & $2,1 \pm 0,79(1,05-4,7)$ & 0.03 \\
\hline \multicolumn{2}{|c|}{$\mathrm{AMH}(\mathrm{pmol} / \mathrm{L})$} & $8,46 \pm 4,87(2,5-24)$ & $2.71 \pm 0.69(1.23-4.11)$ & 0.001 \\
\hline
\end{tabular}


Tab. 3. Two-dimensional ultrasound features of ovaries in terms of antral follicle count and ovarian volume, and comparison of shear wave elastography measures and Doppler indices

\begin{tabular}{|c|c|c|c|}
\hline & $\begin{array}{c}\text { PCOS } \\
\boldsymbol{n}=\mathbf{3 2}\end{array}$ & $\begin{array}{c}\text { non-PCOS } \\
\boldsymbol{n}=\mathbf{3 2}\end{array}$ & $\boldsymbol{p}$ \\
\hline Antral follicle count & $\begin{array}{c}17.61 \pm 3.87 \\
(12-25)\end{array}$ & $\begin{array}{c}9.3 \pm 2.14 \\
(7-12)\end{array}$ & $\mathbf{0 . 0 0 1}$ \\
\hline Mean ovarian volume & $\begin{array}{c}13.26 \pm 5.34 \\
(5.30-29.20)\end{array}$ & $\begin{array}{c}7.23 \pm 1.81 \\
(4.8-11)\end{array}$ & $\mathbf{0 . 0 1}$ \\
\hline PSV & $\begin{array}{c}29.46 \pm 13.75 \\
(11-70)\end{array}$ & $\begin{array}{c}39.47 \pm 15.12 \\
(22-61)\end{array}$ & $\mathbf{0 . 0 0 1}$ \\
\hline RI & $\begin{array}{c}0.76 \pm 0.10 \\
(0.56-0.91)\end{array}$ & $\begin{array}{c}0.71 \pm 0.12 \\
(0.58-0.88)\end{array}$ & $\mathbf{0 . 0 2 1}$ \\
\hline Mean SWV (m/sec) & $\begin{array}{c}2.12 \pm 0.82 \\
(0.78-4.9)\end{array}$ & $\begin{array}{c}1.18 \pm 0.41 \\
(0.77-2.0)\end{array}$ & $\mathbf{0 . 0 1 6}$ \\
\hline \multicolumn{3}{|c|}{ PSV- peak systolic velocity; RI - resistance index; SWV-shear wave velocity } \\
\hline
\end{tabular}

0,001 and $p=0.021$, respectively). Mean SWV of the ovaries were $2.12 \pm 0.82(0.78-4.9)$ in the PCOS group and $1.18 \pm$ $0.41(0.77-2.0)$ in the non-PCOS group $(p=\mathrm{S})$, which was statistically significantly higher in the PCOS women (Tab. 3).

\section{Discussion}

Early application of elasticity measurements in soft tissues to identify tissue hardening due to fibrosis or tumor was first examined in the liver, thyroid and breast tissues ${ }^{(11-13)}$. Elasticity can be measured via ARFI, which is a non-invasive and cost-effective diagnostic tool using the advantages of B-mode ultrasonography. It is generally accepted as a safe and easy method ${ }^{(14)}$.

Tissue elasticity is investigated in various obstetric and gynecologic conditions. In their study, Bildaci et al. used ARFI to evaluate placentas in patients with gestational diabetes, revealing no difference between the patients and the control groups in shear wave velocities. This could be due to the transient pathology of gestational diabetes, since the pathology can improve quickly with dietary regulations on most occasions ${ }^{(15)}$.

Ovarian cysts were evaluated by ARFI in a few studies. In their study, Ciledag et al. found that elastography scores might be useful for differentiating between benign and malignant ovarian cystic lesions. On that basis, unnecessary interventions could be avoided for benign cysts with solid components ${ }^{(16)}$.

PCOS is a result of chronic anovulation. Chronic high levels of androgens cause more theca cells (hence the former name of hyperthecosis); the connective tissue in between follicles is surrounded by androgen-producing cells. Based on the two-cell-two-gonadotropin theory, the hyperandrogenemic environment cannot be beaten due to anovulation, so more androgens are produced, and thus a chronic vicious cycle occurs. This pathologic condition makes the ovarian tissue more stiffer than in normal estrogenic cases.

The diagnosis of PCOS is established based on the 2003 Rotterdam Criteria, after the exclusion of other pathologies, as having 2 of the 3 diagnostic criteria, one of them being oligomenorrhea. In our study, there were significantly prominent menstruation abnormalities in the PCOS group. One criterion is having hyperandrogenism, established either clinically or biochemically. In our study, the PCOS patients were significantly (both clinically and biochemically) hyperandrogeniemic. Another criterion is having polycystic ovarian morphology, i.e. more than 12 antral follicles or more than $10 \mathrm{ml}$ of ovarian volume. In our study, the PCOS patients had a significantly more pronounced polycystic ovarian morphology than the control group.

In their study, Ozdemir et al. compared ovarian stromal blood flow with Doppler ultrasonography in patients with PCOS and healthy controls, revealing that PI was significantly different in the PCOS group ${ }^{(17)}$. In our study, we also found different PI and PSV values in the PCOS group.

In the literature, there are three studies on polycystic ovaries and elastography ${ }^{(18-20)}$. In one of them, the researchers studied the elasticity pattern and strain ratio. Ciraci et al. classified the elasticity patterns of the ovaries in patients with PCOS as hard, medium and soft, and examined the strain rates. They revealed that the elasticity patterns and strain ratios might demonstrate morphological stiffness changes in polycystic ovaries. They also reported that it was possible to evaluate PCOS by real-time elastography, which might help to show morphological changes. Elastography features of ovarian stroma may have a role in the diagnosis of PCOS, like gray-scale US, especially using the strain ratio ${ }^{(18)}$.

In their study, Ertekin et al., reported no significant differences in SWV between the PCOS and control groups. Their conclusion was that the role of elastography in the diagnosis of PCOS was controversial. However, in their study, the sample size of the control group was small, which could have potentially affected the accuracy of their results ${ }^{(19)}$.

In a recent study by Altunkeser et al.; 66 PCOS patients were compared with 72 controls for SWV and elastography values. The authors found that the PCOS group had a mean SWV of $3.89 \pm 1.81$ for the right ovary, and $2.88 \pm 0.65$ for the left ovary. They compared the results with the controls, and found no significant difference ${ }^{(20)}$. Our mean SWV results were $2.12 \pm 0.82(0.78-4.9)$ in the PCOS group, and $1.18 \pm 0.41(0.77-2.0)$ in the control group $(p=0.016)$.

There are different phenotypes in PCOS, so confirming 2 of the 3 criteria is enough for the diagnosis according to the Rotterdam Criteria; women having hyperandrogenism and oligomenorrhea can have the diagnosis without the morphological appearance of $\operatorname{PCOS}^{(21)}$. However; chronic anovulatory condition leads to the hyperandrogenic inner state of the ovaries, resulting in the development of symptoms. SWVs are significantly faster in the ovaries of PCOS patients due to stiffness.

There are women with a normal menstrual pattern, without clinical or biochemical hyperandrogenism, but having 
PCO morphologies seen in two-dimensional US. SWV can be measured in these groups to reveal normal rates with normal ovaries, because they do not have a chronic anovulatory disease pattern.

In our study, all women in the PCOS group had polycystic ovaries resulting from a chronic disease pattern, and none of them had solely PCOS appearance. This is the strength of our study. Limitations include the small of patients studied, and the retrospective design. Consequently, future studies with larger PCOS populations are required for more accurate conclusions.

In our study, we found significantly different SWV values in PCOS patients than non-PCOS women, which indicates that PCOS has an effect on SWV. The increased acoustic frequencies cause a decreased response in time to transition, and motion becomes out of phase; in other words, scattered waves are faster in stiffer ovaries. Our results are thus compatible with the pathophysiology of the disease.

\section{References}

1. Bruno C, Minniti S, Bucci A, Muclli RP: ARFI: from basic principles to clinical applications in diffuse chronic disease - a review. Insights Imaging. 2016; 7: 735-746.

2. Bildaci TB, Cevik H, Yilmaz B, Desteli GA: Value of in vitro acoustic radiation force impulse application on uterine adenomyosis. J Med Ultrason 2018; 45: 425-430.

3. Gao L, Parker KJ, Lerner RM, Levinson SF: Imaging of the elastic properties of tissue - a review. Ultrasound Med Biol 1996; 22: 959-977.

4. Akbas M, Koyuncu FM, Artunç-Ulkumen BA: Placental elasticity assesment by point shear wave elastography in pregnancies with intrauterine growth restriction. J Perinat Med 2019; 27: 841-846.

5. Zhang D-L, Liu X-X, Tang J-Q, Wu S-S, Lin N, Ding G-S et al.: The value of acustic radiation force impulse imaging in preoperative prediction for efficacy of high-intensity focused ultrasound uterine fibroids ablation. Int J Hyperthermia 2020; 37: 423-429.

6. Soliman AA, Wojcinski S, Degenhardt F: Ultrasonographic examination of the endometrium and myometrium using acustic radiation force impulse (ARFI) imaging technology: an initial experience with a new method. Clin Hemorheol Microcirc 2015; 59: 235-243.

7. The Rotterdam ESHRE/ASRM-sponsored PCOS consensus workshop group: Revised 2003 consensus on diagnostic criteria and long term health risks related to polycystic ovary syndrome (PCOS). Hum Reprod 2004; 19: 41-47.

8. Hughesdon PE: Morphology and morphogenesis of the stein-leventhal ovary and the so-called "hyperthecosis". Obstet Gynecol Surv 1982; 37: 59-77.

9. Villarroel C, López P, Merino PM, Iñguinez G, Sir-Petermann T, Codner E: Hirsutism and oligomenorrhea are appropriate screening criteria for polycstic ovary syndrome in adolescents. Gynecol Endocrinol 2015; 31: 625-629.

10. Aswini R, Jayapalan S: Modified Ferriman-Gallweyscore in hirsutism and its association with metabolic syndrome. Int J Trichology 2017; 9: 7-13.

11. Hu X, Huang X, Chen H, Zhang T, Hou J, Song A et al.: Diagnostic effect of shear wave elastography imaging for differentiation of malignant liver lesions: a meta-analysis. BMC Gastroenterol 2019; 19: 60.

\section{Conclusion}

Easy application of quantitative sonoelastography measurements with ARFI provide significant findings about PCOS with regard to shear wave velocity, compatible with chronic anovulation pathophysiology. In light of these findings, SWV is expected to be slower in ovulatory women.

The widespread application of SWV in PCOS diagnosis is not a must, but it can be a beneficial diagnostic tool for clinicians, since shear wave velocities in PCOS patients are found to be significantly faster, reflecting stromal increase and more AFC, which is consistent with the chronic hyperandrogenemic inner environment of the ovarian tissue in PCOS.

\section{Conflict of interest}

Authors do not report any financial or personal connections with other persons or organizations, which might negatively affect the contents of this publication and/or claim authorship rights to this publication.

12. Aghaghazvini L, Maheronnaghsh R, Soltani A, Rouzrokh P, Chavoshi M: Diagnostic value of shear wave sonoelastography in differentiation of benign from malignant thyroid nodules. Eur J Radiol 2020; 126: 108926.

13. Zheng X, Huang Y, Liu Y, Wang Y, Mao R, Li F et al.: Shear-wave elastography of the breast: added value of a quality map in diagnosis and prediction of the biological characteristics of breast cancer. Korean J Radiol 2020; 2: 172-180.

14. Palmeri MI, Frinkley KD, Nightingale KR: Experimental studies on the thermal effects associated with radiation force imaging of soft tissue. Ultrason Imaging 2004; 26: 100-114.

15. Bildacı TB, Çevik H, Aksan Desteli G, Tavaslı B, Özdogan S: Placental elasticity on patients with gestational diabetes: single institution experience. J Chin Med Assoc 2017; 80: 717-720.

16. Ciledag N, Arda K, Aktas E, Aribas BK: A pilot study on real-time transvaginal ultrasonic elastography of cystic ovarian lesions. Indian J Med Res 2013; 137: 1089-1092.

17. Ozdemir O, Sari ME, Kalkan D, Koc M, Ozdemir S, Atalay CR: Comparison of ovarian stromal blood flow measured by color Doppler ultrasonography in polycystic ovary syndrome patients and healthy women with ultrasonographic evidence of polycystic. Gynecol Endocrinol 2015; 31: 322-326.

18. Çıracı S, Tan S, Ozcan AS, Aslan A, Keskin HL, Ateş ÖF et al.: Contribution of real-time elastography in diagnosis of polycystic ovary syndrome. Diagn Interv Radiol 2015; 21(2): 118-122.

19. Ertekin E, Turan OD, Tuncyurek O: Is shear wave elastography relevant in the diagnosis of polycystic ovarian syndrome? Med Ultrason 2019; 21: 158-162.

20. Altunkeser A, Inal ZO, Baran N: Evaluation of ovaries in pateints with polycystic ovary syndrome using shear wave elastography. Curr Med Imaging 2020; 16: 578-583. 